\title{
Reporting Consecutive Substring Occurrences Under Bounded Gap Constraints
}

\author{
Gonzalo Navarro $^{1}{ }^{\star}$ and Sharma V. Thankachan ${ }^{2}$ \\ ${ }^{1}$ Center of Biotechnology and Bioengineering, \\ Department of Computer Science, \\ University of Chile, Chile. \\ gnavarro@dcc.uchile.cl \\ 2 School of Computational Science and Engineering, \\ Georgia Institute of Technology, USA. \\ sharma. thankachan@gatech.edu
}

\begin{abstract}
We study the problem of indexing a text $T[1 \ldots n]$ such that whenever a pattern $P[1 \ldots p]$ and an interval $[\alpha, \beta]$ comes as a query, we can report all pairs $(i, j)$ of consecutive occurrences of $P$ in $T$ with $\alpha \leq j-i \leq \beta$. We present an $O(n \log n)$ space data structure with optimal $O(p+k)$ query time, where $k$ is the output size.
\end{abstract}

\section{Introduction}

Detecting consecutive occurrences of a pattern in a text is a problem that arises, in various forms, in computational biology applications [1$3]$. For example, a tandem repeat is an occurrence of the form $P P$ of a given string $P[1 \ldots p]$ inside a sequence $T[1 \ldots n]$. Due to mutations and experimental errors, one may relax the condition that the occurrences appear exactly one after the other, and allow for a small range of distances between the two occurrences of $P$ [1, Sec. 9.2]. Other variants of the problem are to find $P$ closely followed by its reverse complemented version in tRNA sequences, which is useful to identify the positions where the tRNA molecule folds into a cloverleaf structure defined by stems (the two occurrences of $P$ ) and loops (the string between them) [1, Sec. 11.9, Ex. 42]; this process is also called RNA interference [2, Sec. 6,4].

Several related combinatorial problems stem from these motivations. For example, Iliopoulos and Rahman [4] consider the problem of finding all the $k$ occurrences of two patterns $P_{1}$ and $P_{2}$ (of total length $p$ ) separated by a fixed distance $\alpha$ known at indexing time. They gave a data structure using $O\left(n \log ^{\epsilon} n\right)$ space and query time $O(p+\log \log n+k)$, for any constant $\epsilon>0$. Bille and Gørtz [5] retained the same space

\footnotetext{
* Funded with Basal Funds FB0001, Conicyt, Chile.
} 
and improved the time to the optimal $O(p+k) .{ }^{1}$ The problem becomes, however, much messier when we allow the distance between $P_{1}$ and $P_{2}$ to be in a range $[\alpha, \beta]$, even if these are still known at indexing time. Bille et al. [6] obtained various tradeoffs, for example $O(n)$ space and $O\left(p+\sigma^{\beta} \log \log n+k\right)$ time, where $\sigma$ is the alphabet size; $O\left(n \log n \log ^{\beta} n\right)$ space and $O\left(p+(1+\epsilon)^{\beta} \log \log n+k\right)$ time; and $O\left(\sigma^{\beta^{2}} n \log ^{\beta} \log n\right)$ space and $O((p+\beta)(\beta-\alpha)+k)$ time.

These problems, however, are more general than necessary for the applications we described, where $P_{1}=P_{2}=P$ (or $P_{2}$ is the reverse complement of $P_{1}$, a case that can be handled in the solution we will give). For this case, some related problems have been studied. Keller et al. [7] considered the problem of, given an occurrence of $P$ in $T$, find the next one to the right. They obtained an index using $O\left(n \log ^{\epsilon} n\right)$ space and $O(\log \log n)$ time. Another related problem they studied was to find a maximal set of nonoverlapping occurrences of $P$. They obtained the same space and $O(\log \log n+k)$ time. Muthukrishnan [8] considered a document-based version of the problem: $T$ is divided into documents, and we want to report all the $k$ documents where two occurrences of $P$ appear at distance at most $\beta$. For $\beta$ fixed at indexing time, he obtained $O(n)$ space and optimal $O(p+k)$ time; the space raises to $O(n \log n)$ when $\beta$ is given as a part of the query. Finally, Brodal et al. [9] considered the related pattern mining problem: find the all $z$ maximal patterns $P$ that appear at least twice in $T$, separated by a distance in $[\alpha, \beta]$. They obtain $O(n \log n+z)$ time, within $O(n)$ space.

In this paper we focus on what is perhaps the cleanest variant of the problem, which (somewhat surprisingly) has not been considered before: find the positions in $T$ where two occurrences of $P$ appear, separated by a distance in the range $[\alpha, \beta]$. It is formally stated as follows.

Problem 1 Index a text $T[1 \ldots n]$, such that whenever a pattern $P[1 \ldots p]$ and a range $[\alpha, \beta]$ comes as a query, we can report all pairs $(i, j)$ of consecutive occurrences of $P$ in $T$ with $\alpha \leq j-i \leq \beta$.

We obtain the following result.

Theorem 1 There exists an $O(n \log n)$ space data structure with query time $O(p+k)$ for Problem 1, where $k$ is the output size.

Our solution makes use of heavy-path decompositions on suffix trees and geometric data structures. In the Conclusions we comment on the implications of this result on related problems.

\footnotetext{
${ }^{1}$ This is optimal in the RAM model if we assume a general alphabet of size $O(n)$.
} 


\section{Notation and Preliminaries}

The $i$ th leftmost character of $T$ is denoted by $T[i]$, where $1 \leq i \leq n$. The sub-string starting at location $i$ and ending at location $j$ is denoted by $T[i \ldots j]$. A suffix is a substring that ends at location $n$ and a prefix is a string that starts at location 1 .

The suffix tree (ST) of $T$ is a compact representation of all suffixes of $T \circ \$$, except $\$$, in the form of a compact trie [10]. Here $\$$ a special symbol that does not appear anywhere in $T$ and $T \circ \$$ is the concatenation of $T$ and $\$$. The number of leaves in ST is exactly $n$. The degree of an internal node is at least two. We use $\ell_{i}$ to represent the $i$ th leftmost leaf in ST. The edges are labeled with characters and the concatenation of edge labels on the path from root to a node $u$ is denoted by path $(u)$. Then, path $\left(\ell_{i}\right)$ corresponds to the $i$ th lexicographically smallest suffix of $T$, and its starting position is denoted by SA $[i]$. The locus of a pattern $P$ in $T$, denoted by locus $(P)$, is the highest node $u$ in $\mathrm{ST}$, such that $P$ is a prefix of path $(u)$. The set of occurrences of $P$ in $T$ is given by SA $[i]$ over all $i$ 's, where $\ell_{i}$ is in the subtree of locus $(P)$. The space occupied by ST is $O(n)$ words and the time for finding the locus of an input pattern $P$ is $O(|P|)$. Additionally, for two nodes $u$ and $v$, we shall use $\operatorname{Ica}(u, v)$ to denote their lowest common ancestor.

We now describe the concept of heavy path and heavy path decomposition. The heavy path of ST is the path starting from the root, where each node $u$ on the path is the child with the largest subtree size (ties broken arbitrary). The heavy path decomposition is the operation where we decompose each off-path subtree of the heavy path recursively. As a result, any path $(\cdot)$ in ST will be partitioned into disjoint heavy paths. Sleator and Tarjan [11] proved the following property; we will use $\log n$ to denote logarithm in base 2 .

Lemma 1 The number of heavy paths intersected by any root to leaf path is at most $\log n$, where $n$ is the number of leaves in the tree.

Each node belongs to exactly one heavy path and each heavy path contains exactly one leaf node. The heavy path containing $\ell_{i}$ will be called the $i$-th heavy path (and identified simply by the number $i$ ). For an internal node $u$, let $h p(u)$ be the unique heavy path that contains $u$.

Definition 1 The set $\mathcal{H}_{i}$ is defined as the set of all leaf identifiers $j$, where the path from root to $\ell_{j}$ intersects with the $i$-th heavy path. That $i s, \mathcal{H}_{i}=\left\{j \mid \operatorname{hp}\left(\operatorname{Ica}\left(\ell_{j}, \ell_{i}\right)\right)=i\right\}$. 
Lemma $2 \sum_{i=i}^{n}\left|\mathcal{H}_{i}\right| \leq n \log n$.

Proof. For any particular $j$, path from root to $\ell_{j}$ can intersect at most $\log n$ heavy paths, by Lemma 1 . Therefore, $j$ cannot be a part of more than $\log n$ sets.

\section{The Data Structure}

The key idea is to reduce our pattern matching problem to an equivalent geometric problem. Specifically, to the orthogonal segment intersection problem.

Definition 2 (Orthogonal Segment Intersection) A horizontal segment $\left(x_{i}, x_{i}^{\prime}, y_{i}\right)$ is a line connecting the $2 D$ points $\left(x_{i}, y_{i}\right)$ and $\left(x_{i}^{\prime}, y_{i}\right)$. A segment intersection problem asks to pre-process a given set $\mathcal{S}$ of horizontal segments into a data structure, such that whenever a vertical segment $\left(x^{\prime \prime}, y^{\prime}, y^{\prime \prime}\right)$ comes as a query, we can efficiently report all the horizontal segments in $\mathcal{S}$ that intersect with the query segment. Specifically, we can output the following set: $\left\{\left(x_{i}, x_{i}^{\prime}, y_{i}\right) \in \mathcal{S} \mid x_{i} \leq x^{\prime \prime} \leq x_{i}^{\prime}, y^{\prime} \leq y_{i} \leq y^{\prime \prime}\right\}$.

There exists an $O(|\mathcal{S}|)$ space and $O(\log |\mathcal{S}|+k)$ time solution for segment intersection problem using a persistent binary tree, where $k$ is the output size [12]. We now proceed to describe the reduction.

\section{$3.1 \quad$ Reduction}

One of the main components of our data structure is the suffix tree ST of $T$, and is used only for finding the locus of $P$. Based on the heavy path on which the locus node is, we categorize the queries in different types.

Definition 3 A query with input pattern $P$ is type-h if $h=\mathrm{hp}(\operatorname{locus}(P))$.

Let $G_{h}$ be the data structure handling type- $h$ queries, where $G_{h}$ is a structure over a set $\mathcal{I}_{h}$ of horizontal segments, that can efficiently answer segment intersection queries. The set $\mathcal{I}_{h}$ is generated from $\mathcal{H}_{h}$ using the following steps for each $j \in \mathcal{H}_{h}$ :

1. Let $P_{j}=\operatorname{path}\left(\operatorname{Ica}\left(\ell_{h}, \ell_{j}\right)\right)$

2. Let $\operatorname{suc}(j)$ be the first occurrence of $P_{j}$ after the position $\mathrm{SA}[j]$ in $T$ and let $\operatorname{pre}(j)$ be the last occurrence of $P_{j}$ before the position $\mathrm{SA}[j]$ in $T$. Clearly, neither in $[(\operatorname{pre}(j)+1) \ldots(\mathrm{SA}[j]-1)]$, nor in $[(\mathrm{SA}[j]+1) \ldots(\operatorname{suc}(j)-1)], P_{j}$ has an occurrence. 


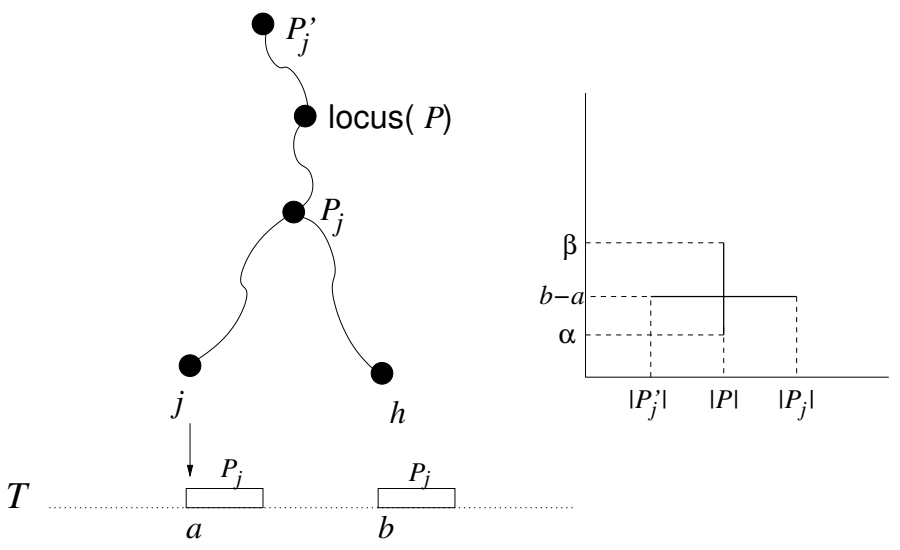

Fig. 1. Illustration of the main concepts of our data structure.

3. Now, obtain two segments w.r.t. $j$ as follows:

(a) Let $P_{j}^{\prime}$ be the shortest prefix of $P_{j}$ without any occurrence in $[(\operatorname{pre}(j)+1) \ldots(\mathrm{SA}[j]-1)]$. Then, create segment $\left(x_{i}, x_{i}^{\prime}, y_{i}\right)=$ $\left(\left|P_{j}^{\prime}\right|,\left|P_{j}\right|, \mathrm{SA}[j]-\operatorname{pre}(j)\right)$ and associate the pair $(\operatorname{pre}(j), \mathrm{SA}[j])$ of consecutive occurrences of $P_{j}$ as satellite information.

(b) Similarly, let $P_{j}^{\prime \prime}$ be the shortest prefix of $P_{j}$ without any occurrence in $[(\mathrm{SA}[j]+1) \ldots(\operatorname{suc}(j)-1)]$. Then, create segment $\left(x_{i}, x_{i}^{\prime}, y_{i}\right)=\left(\left|P_{j}^{\prime \prime}\right|,\left|P_{j}\right|, \operatorname{suc}(j)-\mathrm{SA}[j]\right)$ and associate it to the pair $(\mathrm{SA}[j], \operatorname{suc}(j))$ of consecutive occurrences of $P_{j}$ as satellite information.

Clearly, $\left|\mathcal{I}_{h}\right|=2\left|\mathcal{H}_{h}\right|$. The central idea of our solution is summarized below. Figure 1 illustrates the idea.

Lemma 3 Let $P$ and $[\alpha, \beta]$ be the input parameters of a query in problem 1 and let $h=\mathrm{hp}(\operatorname{locus}(P))$. Then, the set of satellite information associated with all those horizontal segments in $\mathcal{I}_{h}$, which are stabbed by a vertical segment $(p, \alpha, \beta)$ (i.e., the segment connecting the points $(p, \alpha)$ and $(p, \beta))$ forms the output to Problem 1 .

Proof. First we prove that any satellite information $(a, b)$ reported by the geometric query on $G_{h}$ is an answer to the original query. Let $[s, e]$ be the $x$-interval corresponding to the reported satellite information $(a, b)$. Then, $s \leq p \leq e$ and $\alpha \leq b-a \leq \beta$. Here the condition $e \geq p$ ensures that both $\ell_{\mathrm{SA}^{-1}[a]}$ and $\ell_{\mathrm{SA}^{-1}[b]}$ are leaves in the subtree of locus $(P)$. Therefore $a$ and $b$ are occurrences of $P$. The condition $s \leq p$ ensures that there 
exists no occurrence of $P$ in any location which is after $a$, but before $b$ (i.e., $a$ and $b$ are consecutive occurrences of $P$ ). Finally the $y$-coordinate ensures that $\alpha \leq b-a \leq \beta$.

Now we prove that for every output $(a, b)$ of Problem 1 , there exists a segment $(s, e, b-a)$ in $\mathcal{I}_{h}$ with $s \leq p \leq e$ and satellite information $(a, b)$. Without loss of generality, let $\operatorname{Ica}\left(\ell_{h}, \ell_{\mathrm{SA}}{ }^{-1}[a]\right)$ be either $\operatorname{Ica}\left(\ell_{h}, \ell_{\mathrm{SA}^{-1}[b]}\right)$ or an ancestor of it. Then, let $j=\mathrm{SA}^{-1}[a]$. Since $P$ occurs at position $a$, the leaf $j$ descends from the subtree of locus $(P)$, and since this node belongs to the heavy path $h$, we have that Ica $\left(\ell_{h}, \ell_{j}\right)$ descends from locus $(P)$, thus $e \geq p$. Since there is no occurrence of $P$ between $a$ and $b$, it holds $s \leq p$. Then, a segment of the form $(s, e, b-a)$ will indeed be created while processing $j \in \mathcal{H}_{h}$ during the construction of $\mathcal{I}_{h}$.

In the light of Lemma 3, we have the following result.

Lemma 4 There exists an $O(n \log n)$ space and $O(p+\log n+k)$ query time solution for Problem 1, where $k$ is the output size.

Proof. The space of ST is $O(n)$ and the space required for maintaining the segment intersection structure over $\mathcal{I}_{h}$, for all values of $h$, is $O\left(\sum_{h}\left|\mathcal{I}_{h}\right|\right)=$ $O\left(\sum_{h}\left|\mathcal{H}_{h}\right|\right)=O(n \log n)$. Thus, the total space is $O(n \log n)$ words. To answer a query, we first find the locus of $P$ in ST in $O(p)$ time, and then query $G_{h}$, where $h=\mathrm{hp}(\operatorname{locus}(P))$, in $O(\log n+k)$ time. Therefore, the query time is $O(p+\log n+k)$.

The query time in Lemma 4 is optimal if $p \geq \log n$. To handle queries where $p$ is shorter than $\log n$, we use a different approach.

\subsection{Achieving Optimal Query Time}

We present an optimal query time data structure for $p<\log n$. Essentially, we associate a data structure $D(u)$ with each node $u$ in ST, whose string depth (i.e., $|\operatorname{path}(u)|)$ is at $\operatorname{most} \log n$. Observe that the number of occurrences of path $(u)$ in $T$ is equal to $\operatorname{size}(u)$, where $\operatorname{size}(u)$ is the number of leaves in the subtree of $u$. Therefore, the number of consecutive occurrences $(i, j)$ of $\operatorname{path}(u)$ is $\operatorname{size}(u)-1$. Each such pair $(i, j)$ can be mapped to a point $(j-i)$ in one dimension along with the pair $(i, j)$ as an associated satellite data. We then create a one-dimensional range reporting data structure over these $(\operatorname{size}(u)-1)$ points and call it $D(u)$. Whenever the locus of $P$ is $u$, the answer can be obtained by issuing a one dimensional range reporting query on $D(u)$ with $[\alpha, \beta]$ as the input 
range. The satellite data associated with each reported corresponds to an answer to Problem 1.

We use the data structure summarized in Lemma 5 , by which queries can be answered in optimal time and the space of $D(u)$ can be bounded by $O(\operatorname{size}(u))$ words.

Lemma 5 ([13]) One dimensional range reporting queries over a set of $m$ points in $\left\{0,1,2, \ldots, 2^{w}\right\}$ can be answered in optimal time using an $O(m)$ space data structure, where $w$ is the word size.

Note that the sum of all the $\operatorname{size}(u)$ terms for all the nodes $u$ with the same string depth is $n$, and added over all the nodes with string depth up to $\log n$ is $n \log n$. Thus the space for the $D(\cdot)$ structures of all the nodes with string depth up to $\log n$ is $O(n \log n)$ words. This completes the proof of Theorem 1 .

\section{Conclusions}

We have addressed what seems to be the cleanest variant of the problem related to finding close occurrences of a pattern $P[1 \ldots p]$ in a text $T[1 \ldots n]$ : find pairs of occurrences that are within a distance range $[\alpha, \beta]$ (given at query time). Our data structure uses $O(n \log n)$ space and optimal $O(p+k)$ query time.

It is not hard to extend our result to the case where we look for the occurrence of $P$ followed (or preceded) by some function of $P$, such as its reverse complemented string (as motivated in the Introduction). We can build the geometric structure at each suffix tree node $v$ considering the function of the string represented by $v$, instead of the string itself. However, extending our solution to the general case of two patterns [6] seems not possible.

Our result opens several interesting questions. A first one is whether this problem is strictly harder than the restricted variant where $\alpha=\beta$. For this case, the same optimal query time has been obtained within less space, $O\left(n \log ^{\epsilon} n\right)$ [5], even when generalizing the problem to two patterns $P_{1}$ and $P_{2}$. The significantly messier results obtained for the general case $\alpha \leq \beta$ [6] suggest that this general problem is indeed harder. Still, it is not clear whether our optimal-time result can also be obtained within $o(n \log n)$ space.

A second interesting question is whether our result can be used for pattern mining, that is, finding those $P$ that appear twice in $T$ separated by a distance in $[\alpha, \beta]$. A direct application of our result, which builds 
our structure and then traverses the suffix tree, requires $\Omega(n \log n+z)$ time, which is not better than the current result [9]. Yet, there could be harder pattern mining problems for which our result is a useful tool.

Yet a third interesting question is how our results can be extended to the document retrieval scenario, that is, listing the documents where $P$ appears twice and separated by a distance in $[\alpha, \beta]$. The current result [8] is similar to ours in space and time, but it is restricted to the case $\alpha=0$. It is not clear if is the problem is harder, and by how much, for an arbitrary value of $\alpha$.

\section{References}

1. Gusfield, D.: Algorithms on Strings, Trees and Sequences: Computer Science and Computational Biology. Cambridge University Press (1997)

2. Aluru, S., ed.: Handbook of Computational Molecular Biology. CRC Computer and Information Science Series. Chapman \& Hall (2005)

3. Ohlebusch, E.: Bioinformatics Algorithms: Sequence Analysis, Genome Rearrangements, and Phylogenetic Reconstruction. Oldenbusch Verlag (2013)

4. Iliopoulos, C.S., Rahman, M.S.: Indexing factors with gaps. Algorithmica 55 (2009) 60-70

5. Bille, P., Gørtz, I.L.: Substring range reporting. Algorithmica 69 (2014) 384-396

6. Bille, P., Gørtz, I.L., Vildhøj, H.W., Vind, S.: String indexing for patterns with wildcards. Theory of Computing Systems 55 (2014) 41-60

7. Keller, O., Kopelowitz, T., Lewenstein, M.: Range non-overlapping indexing and successive list indexing. In: Proc. 10th International Workshop on Algorithms and Data Structures (WADS). LNCS 4619 (2007) 625-636

8. Muthukrishnan, S.: Efficient algorithms for document retrieval problems. In: Proc 13th Annual ACM-SIAM Symposium on Discrete Algorithms (SODA). (2002) 657666

9. Brodal, G.S., Lyngs $\varnothing$, R.B., Pedersen, C.N.S., Stoye, J.: Finding maximal pairs with bounded gap. In: Proc. 10th Annual Symposium on Combinatorial Pattern Matching (CPM). LNCS 1645 (1999) 134-149

10. Weiner, P.: Linear pattern matching algorithms. In: 14th Annual Symposium on Switching and Automata Theory, Iowa City, Iowa, USA, October 15-17, 1973. (1973) 1-11

11. Sleator, D.D., Tarjan, R.E.: A data structure for dynamic trees. J. Comput. Syst. Sci. 26 (1983) 362-391

12. Tao, Y.: Dynamic ray stabbing. ACM Transactions on Algorithms 11 (2014) 11

13. Alstrup, S., Brodal, G.S., Rauhe, T.: Optimal static range reporting in one dimension. In: Proceedings on 33rd Annual ACM Symposium on Theory of Computing, July 6-8, 2001, Heraklion, Crete, Greece. (2001) 476-482 